ANNALES

POLONICI MATHEMATICI

$95.3(2009)$

\title{
Dynamical systems method for solving linear ill-posed problems
}

\author{
by A. G. RAmm (Manhattan, KS)
}

\begin{abstract}
Various versions of the Dynamical Systems Method (DSM) are proposed for solving linear ill-posed problems with bounded and unbounded operators. Convergence of the proposed methods is proved. Some new results concerning the discrepancy principle for choosing the regularization parameter are obtained.
\end{abstract}

1. Introduction. In this paper we present part of the results from the author's invited plenary talk at the international conference on mathematical analysis and applications ICMAAS06, held in Egypt. This part deals with linear ill-posed problems.

Some of the ideas and results in this paper are taken from the papers of the author, cited in the bibliography, but many results are new, including Theorems 2-8 and 10.

The Dynamical Systems Method (DSM) is developed in [7], [8], [9]-[33]. The discrepancy principle was discussed earlier in [5]. Its analogs and new versions have been studied recently in [9]-[11] and in [7], [8] for DSM.

Consider an equation

$$
A u-f=0,
$$

where $A$ is an operator in a Banach space $X$. If $A$ is a homeomorphism of $X$ onto $X$ (i.e., a continuous injective and surjective map in $X$ which has a continuous inverse) then problem (1.1) is called well-posed in the Hadamard sense. Otherwise it is called ill-posed.

The DSM for solving equation (1.1) consists of solving the Cauchy problem

$$
\dot{u}=\Phi(t, u), \quad u(0)=u_{0}, \quad \dot{u}=\frac{d u}{d t},
$$

2000 Mathematics Subject Classification: 47A52, 47B25, 65R30.

Key words and phrases: ill-posed problems, dynamical systems method (DSM), regularization parameter, discrepancy principle, unbounded operators, linear operator equations. 
which we call a dynamical system, where $\Phi$ is chosen so that the problem (1.2) has a unique solution $u(t)$, defined for all $t \geq 0$, and such the limit $u(\infty):=\lim _{t \rightarrow \infty} u(t)$ exists and satisfies $A(u(\infty))=f$ :

$$
\exists ! u(t) \forall t \geq 0 ; \quad \exists u(\infty) ; \quad A(u(\infty))=f .
$$

We do not assume that the solution to (1.1) is unique, but we do assume that it exists. If (1.3) holds, then we say that DSM is justified for solving equation (1.1).

There is a large body of literature on solving ill-posed problems (see, e.g., [2], [7], [39] and references therein). Variational regularization, iterative regularization, quasisolutions and quasiinversion are some of the methods for stable solution of ill-posed problems discussed in the literature. In this paper several new methods for stable solution of linear ill-posed problems are discussed. They are based on the Dynamical Systems Method. This method has been developed fairly recently for solving a wide variety of linear and nonlinear ill-posed problems [9]-[38] although it was proposed already in [1] for solving well-posed problems.

Because of space limitations we will not discuss solving nonlinear illposed problems by the DSM, and refer the reader to [7], [8], [17], [33]-[38].

Here we describe a new version of DSM for solving linear ill-posed problems. There are many practical problems of this type. We only mention solving ill-conditioned linear algebraic systems and Fredholm equations of the first kind ([32], [33]). The novel points in our results are not only the method of solving these problems by DSM but also the applicability of the method to unbounded operators ([20], [22]-[23], [25]). In the literature, a widelydiscussed method for solving ill-posed problems (1.1) is the method of variational regularization introduced by Phillips [6] and studied by Tikhonov [39], Morozov [5], Ivanov [2], Ramm ([7], [8]) and many other authors under the assumption that the operator $A$ in (1.1) is a linear bounded operator. There are also some results on regularization of unbounded operators (e.g. [4], [28] [20]-[22], [25]). The variational regularization method for stable solution of (1.1) consists in solving the problem

$$
F(u):=\left\|A u-f_{\delta}\right\|^{2}+a\|u\|^{2}=\min ,
$$

where $a>0$ is a constant, called a regularization parameter, $f_{\delta}$ is the "noisy data", i.e., an element which satisfies the inequality $\left\|f_{\delta}-f\right\| \leq \delta$ and which is given together with the "noise level" $\delta>0$, while the exact data $f$ is not known. A stable solution to (1.1) is an element $u_{\delta}$ such that $\lim _{\delta \rightarrow 0}\left\|u_{\delta}-y\right\|$ $=0$, where $A y=f$ and $y$ is the unique minimal-norm solution of the linear equation (1.1). If $X$ is a Hilbert space $H$, which we assume below, then the minimal-norm solution is the solution which is orthogonal to the null space $N$ of $A, N=N(A)=\{u: A u=0\}$. If the linear operator $A$ in (1.1) is 
unbounded, then we assume that it is closed and densely defined, so that its adjoint $A^{*}$ is densely defined and closed (see, e.g., [3]). The DSM for such operators is developed in [20]-[22], [25], [28], [33], and [8].

If $A$ is bounded, then a necessary and sufficient condition for $u$ to be the minimizer of the quadratic functional (1.4) is the Euler equation

$$
T_{a} u=A^{*} f_{\delta}, \quad T_{a}:=T+a I, T:=A^{*} A,
$$

where $I$ is the identity operator and $T \geq 0$ is a selfadjoint operator. Equation (1.5) has a unique solution $u_{a, \delta}=T_{a}^{-1} A^{*} f_{\delta}$. One can choose $a=a(\delta)$ so that $\lim _{\delta \rightarrow 0} a(\delta)=0$ and $u_{\delta}:=u_{a(\delta), \delta}$ is a stable solution to (1.1):

$$
\lim _{\delta \rightarrow 0}\left\|u_{\delta}-y\right\|=0,
$$

where $A y=f$ and $y \perp N$. There are a priori choices of $a(\delta)$ and a posteriori ones. An a priori choice is based on the estimate

$$
\begin{aligned}
\left\|T_{a}^{-1} A^{*} f_{\delta}-y\right\| & \leq\left\|T_{a}^{-1} A^{*}\left(f_{\delta}-f\right)\right\|+\left\|T_{a}^{-1} A^{*} f-y\right\| \\
& \leq \frac{\delta}{2 \sqrt{a}}+\eta(a),
\end{aligned}
$$

where

$$
\eta^{2}(a)=\left\|T_{a}^{-1} T y-y\right\|^{2}=a^{2} \int_{0}^{\infty} \frac{d\left(E_{s} y, y\right)}{(s+a)^{2}} \rightarrow\left\|P_{N} y\right\|^{2} \quad \text { as } a \rightarrow 0,
$$

and $P_{N}$ is the orthoprojector onto $N$. Since we assume that $y \perp N$, equation (1.8) implies

$$
\lim _{a \rightarrow 0} \eta(a)=0 .
$$

The term $\delta /(2 \sqrt{a})$ in (1.7) appears due to the estimate

$$
\begin{aligned}
\left\|T_{a}^{-1} A^{*}\right\| & =\left\|A^{*} Q_{a}^{-1}\right\|=\left\|U Q^{1 / 2} Q_{a}^{-1}\right\| \\
& \leq\left\|Q^{1 / 2} Q_{a}^{-1}\right\|=\sup _{s \geq 0} \frac{\sqrt{s}}{s+a}=\frac{1}{2 \sqrt{a}} .
\end{aligned}
$$

Here $Q:=A A^{*}, U$ is a partial isometry, $Q_{a}:=Q+a I$, and we have used the formula

$$
T_{a}^{-1} A^{*}=A^{*} Q_{a}^{-1},
$$

the polar decomposition $A^{*}=U Q^{1 / 2}$, and the spectral theorem for the selfadjoint operator $Q$, namely $\|g(Q)\|=\sup _{s \geq 0}|g(s)|$. Formula (1.11) is obvious if $A$ is bounded: multiply (1.11) by $T_{a}$ on the left and then by $Q_{a}$ on the right, and get $A^{*}\left(A A^{*}+a I\right)=\left(A^{*} A+a I\right) A^{*}$, which is an obvious identity. Since the operators $Q_{a}$ and $T_{a}$ are boundedly invertible, one may reverse steps and get (1.11). Thus a priori choices of $a(\delta)$, which imply (1.6), 
are

$$
\lim _{\delta \rightarrow 0} \frac{\delta}{\sqrt{a(\delta)}}=0, \quad \lim _{\delta \rightarrow 0} a(\delta)=0 .
$$

There are many functions $a(\delta)$ satisfying (1.12). One can find an optimal value $a(\delta)$ by minimizing the right-hand side of (1.7) with respect to $a$. Alternatively, one may calculate $a(\delta)$ by solving the equation $\delta=2 \sqrt{a(\delta)} \eta(a)$ for $a$ for a fixed small $\delta>0$.

If $A$ is closed, densely defined in $H$, unbounded, and $a=$ const $>0$, then the author has proved in [22] that the operator $T_{a}^{-1} A^{*}$, with the domain $D\left(A^{*}\right)$, is closable, its closure, denoted again $T_{a}^{-1} A^{*}$, is a bounded operator defined on all of $H,\left\|T_{a}^{-1} A^{*}\right\| \leq 1 /(2 \sqrt{a})$, and (1.1) holds.

For convenience of the reader let us sketch the proof of these claims. To check that $T_{a}^{-1} A^{*}$ is closable, one takes $h_{n} \in D\left(T_{a}^{-1} A^{*}\right)=D\left(A^{*}\right)$ such that $h_{n} \rightarrow 0$ and $T_{a}^{-1} A^{*} h_{n} \rightarrow g$ as $n \rightarrow \infty$, and checks that $g=0$. Indeed, let $u \in H$ be arbitrary. Then

$$
(g, u)=\lim _{n \rightarrow \infty}\left(T_{a}^{-1} A^{*} h_{n}, u\right)=\lim _{n \rightarrow \infty}\left(h_{n}, A T_{a}^{-1} u\right)=0 .
$$

Since $u$ is arbitrary, this implies $g=0$, as claimed. Note that $T_{a}^{-1} u \in D(A)$, so that the above calculation is justified. If one drops the index $n$ and the $\lim$ in (1.13), then one can see that the adjoint to the closure of $T_{a}^{-1} A^{*}$ is the operator $A T_{a}^{-1}$, defined on all of $H$ and bounded:

$$
\left\|A T_{a}^{-1}\right\|=\left\|U T^{1 / 2} T_{a}^{-1}\right\| \leq \frac{1}{2 \sqrt{a}} .
$$

Since $\left\|A^{*}\right\|=\|A\|$, one gets $\left\|T_{a}^{-1} A^{*}\right\| \leq 1 /(2 \sqrt{a})$. Finally, formula (1.11) can be proved for an unbounded closed, densely defined operator $A$ as above, if one checks that the operator $A^{*} A A^{*}$ is densely defined. This is indeed the case, because the operator $A^{*} A A^{*} A=T^{2}$ is densely defined if $T$ is, and $D\left(T^{2}\right) \subset D\left(A^{*} A A^{*}\right)$.

Let us now describe an a posteriori choice of $a(\delta)$ which implies (1.6) and which is called the discrepancy principle. This principle was discussed in [5], [7]. It consists in finding $a(\delta)$ from the equation

$$
\left\|A u_{a, \delta}-f_{\delta}\right\|=C \delta, \quad 1<C<2,
$$

where $C=$ const, $\left\|f_{\delta}\right\|>C \delta$ and $u_{a, \delta}=T_{a}^{-1} A^{*} f_{\delta}$. One can prove (see e.g. [7]) that equation (1.14) for a small fixed $\delta>0$ has a unique solution $a(\delta)$ with $\lim _{\delta \rightarrow 0} a(\delta)=0$ and $u_{\delta}=u_{a(\delta), \delta}$ satisfies (1.6), i.e. $u_{\delta}$ is a stable solution to (1.1). To prove these claims one denotes by $P_{N}$ the orthogonal 
projection onto a subspace $N$, writes (1.14) as

$$
\begin{aligned}
C^{2} \delta^{2} & =\left\|\left[A T_{a}^{-1} A^{*}-I\right] f_{\delta}\right\|^{2}=\left\|\left[Q Q_{a}^{-1}-I\right] f_{\delta}\right\|^{2} \\
& =\int_{0}^{\infty} \frac{a^{2} d\left(E_{\delta} f_{\delta}, f_{\delta}\right)}{(s+a)^{2}}=: h(a, \delta),
\end{aligned}
$$

and takes into account that $h(a, \delta)$ is, for a fixed $\delta>0$, a continuous monotone function of $a$, with $h(\infty, \delta)=\left\|f_{\delta}\right\|^{2}>C^{2} \delta^{2}$ and $h(+0, \delta)=\left\|P_{N^{*}} f_{\delta}\right\|$ $\leq \delta^{2}$, so that there exists a unique $a=a(\delta)$ such that $h(a(\delta), \delta)=C^{2} \delta^{2}$. Here we have set $N^{*}:=N\left(A^{*}\right)$ and used the obvious relation $N(Q)=N\left(A^{*}\right)$, the inequality $\left\|P_{N^{*}} f_{\delta}\right\| \leq\left\|P_{N^{*}}\left(f_{\delta}-f\right)\right\|+\left\|P_{N^{*}} f\right\| \leq\left\|f_{\delta}-f\right\|=\delta$, and the relation $P_{N^{*}} f=0$. This last relation follows from the relations $f \in R(A)$ and $R(A) \perp N^{*}$.

Let us now check that if $a(\delta)$ solves (1.5) then $u_{\delta}=u_{a(\delta), \delta}$ satisfies (1.6). One has $F\left(u_{\delta}\right) \leq F(y)$, so

$$
\left\|A u_{\delta}-f_{\delta}\right\|^{2}+a(\delta)\left\|u_{\delta}\right\|^{2} \leq \delta^{2}+a(\delta)\|y\|^{2} .
$$

Since $\left\|A u_{\delta}-f_{\delta}\right\|^{2}=C^{2} \delta^{2}>\delta^{2}$ and $a(\delta)>0$, one gets

$$
\left\|u_{\delta}\right\| \leq\|y\| \text {. }
$$

Therefore one can select a weakly convergent sequence $u_{n}=u_{\delta_{n}} \rightarrow u$ as $n \rightarrow \infty$. Let us prove that $u=y$ and $\lim _{n \rightarrow \infty}\left\|u_{n}-y\right\|=0$. Since this holds for any subsequence, it will then follow that (1.6) holds. To prove that $u=y$ note that (1.17) implies $\|u\| \leq\|y\|$, and that $u$ solves (1.1). Since the minimal-norm solution to (1.1) is unique, it follows that $u=y$. To check that $u$ solves (1.1) we note that $\lim _{\delta \rightarrow 0}\left\|A u_{\delta}-f\right\|=0$, as follows from (1.16) because $\lim _{\delta \rightarrow 0} a(\delta)=0$. The relations $u_{\delta} \rightarrow u$ and $\left\|A u_{\delta}-f\right\| \rightarrow 0$ as $\delta \rightarrow 0$ imply $A u=f$ and $\lim _{\delta \rightarrow 0}\left\|u_{\delta}-u\right\|=0$. Indeed, let us first check that $A u=f$. One has

$$
(f, g)=\lim _{\delta \rightarrow 0}\left(A u_{\delta}, g\right)=\left(u, A^{*} g\right) \quad \forall g \in D\left(A^{*}\right) .
$$

Thus $u \in D(A)$ and $A u=f$, as claimed. As proved above, this implies that $u=y$. Therefore $u_{\delta} \rightarrow y$ and $\left\|u_{\delta}\right\| \leq\|y\|$. This implies that $\lim _{\delta \rightarrow 0}\left\|u_{\delta}-y\right\|=0$. Indeed,

$$
\begin{aligned}
\left\|u_{\delta}-y\right\|^{2} & =\left\|u_{\delta}\right\|^{2}+\|u\|^{2}-2 \operatorname{Re}\left(u_{\delta}, y\right) \\
& \leq 2\|y\|^{2}-2 \operatorname{Re}\left(u_{\delta}, y\right) \rightarrow 0 \quad \text { as } \delta \rightarrow 0 .
\end{aligned}
$$

Thus, the relation (1.6) is proved for the choice of $a(\delta)$ by the discrepancy principle.

The drawback of the a priori choice of $a(\delta)$ is that it is nonunique and although it guarantees convergence (1.16), the error of the method can be large if $\delta>0$ is fixed. The drawback of the discrepancy principle is the 
necessity of solving the nonlinear equation (1.14) and also a possible large error for a fixed $\delta$.

In Section 2 we discuss the DSM for solving linear equations (1.1).

2. DSM for solving linear problems. We assume first that the linear closed densely defined in $H$ operator in (1.1) is selfadjoint, $A=A^{*}$. This is not an essential restriction: every solvable linear equation (1.1) is equivalent to the equation $T u=A^{*} f$, where $T=T^{*}=A^{*} A$. Indeed, if $A u=f$, then applying $A^{*}$ and assuming $f \in D\left(A^{*}\right)$, one gets $T u=A^{*} f$. Conversely, if $T u=A^{*} f$ and $f=A y$, then $T u=T y$. Multiply the equation $0=T(u-y)$ by $u-y$ to get $0=\left(A^{*} A(u-y), u-y\right)=\|A u-A y\|^{2}$. Thus $A u=A y=f$. If $A$ is bounded, then $f \in D\left(A^{*}\right)$ for any $f \in H$. If $A$ is unbounded, then $D\left(A^{*}\right)$ is a linear dense subset of $H$. In this case, if $f \notin D\left(A^{*}\right)$, then we define a solution of the equation $T u=A^{*} f$ by the formula $u=\lim _{a \rightarrow 0} T_{a}^{-1} A^{*} f$. As we have proved in Section 1, for any $f \in R(A)$ this limit exists and equals the minimal-norm solution $y$ : $\lim _{a \rightarrow 0} T_{a}^{-1} A^{*} A u=y$ if $A u=f$. This is true because $\lim _{a \rightarrow 0} T_{a}^{-1} T u=u-P_{N} u=y$.

The DSM for solving equation (1.1) with a linear selfadjoint operator can be constructed as follows. Consider the problem

$$
\dot{u}_{a}=i(A+i a) u_{a}-i f, \quad u(0)=0 ; \quad \dot{u}=\frac{d u}{d t},
$$

where $a=$ const $>0$. Our first result is formulated as Theorem 1 .

Theorem 1. If $A y=f$ and $y \perp N$, then

$$
\lim _{a \rightarrow 0} \lim _{t \rightarrow \infty} u_{a}(t)=y .
$$

Our second result shows that the method, based on Theorem 1, gives a stable solution of the equation $A u=f$. Assume that $\left\|f_{\delta}-f\right\| \leq \delta$, and let $u_{a, \delta}(t)$ be the solution to $(2.1)$ with $f_{\delta}$ in place of $f$.

TheOREM 2. There exist $t=t_{\delta}$ with $\lim _{\delta \rightarrow 0} t_{\delta}=\infty$, and $a=a(\delta)$ with $\lim _{\delta \rightarrow 0} a(\delta)=0$, such that $u_{\delta}:=u_{a(\delta), \delta}\left(t_{\delta}\right)$ satisfies (1.6).

We will discuss the ways to choose $a(\delta)$ and $t_{\delta}$ after the proofs of these theorems are given.

From the numerical point of view, if one integrates problem (2.1) with the exact data $f$ on the interval $0 \leq t \leq T$, and $T$ is fixed, then one is interested in choosing $a=a(T)$ such that $\lim _{T \rightarrow \infty}\left\|u_{a(T)}(T)-y\right\|=0$. We will give such a choice of $a(T)$.

Before we start proving these two theorems, let us explain the ideas of the proof. Suppose $B$ is a linear operator and its inverse $B^{-1}$ exists and is bounded. 
Finally, assume that

$$
\lim _{t \rightarrow \infty}\left\|e^{B t}\right\|=0 .
$$

This will happen, for example, if $\operatorname{Re} B \leq-c$, where $c>0$ is a constant. Under these assumptions one has

$$
\int_{0}^{t} e^{B s} d s=B^{-1}\left(e^{B t}-I\right), \quad-\lim _{t \rightarrow \infty} \int_{0}^{t} e^{B s} d s=B^{-1} .
$$

The operator $\int_{0}^{t} e^{B s} d s$ solves the problem

$$
\dot{W}=B W+I, \quad W(0)=0,
$$

where $I$ is the identity operator. If $\lim _{t \rightarrow \infty}\left\|e^{B t}\right\|=0$, then

$$
-\lim _{t \rightarrow \infty} W(t)=B^{-1} \text {. }
$$

The basic idea of the DSM is the representation of the inverse operator as the limit as $t \rightarrow \infty$ of the solution to the Cauchy problem (2.5).

Proof of Theorem 1. The solution to (2.1) is

$$
u_{a}(t)=\int_{0}^{t} e^{i(A+i a)(t-s)}(i f) d s=[i(A+i a)]^{-1}\left(e^{i(A+i a) t}-I\right)(-i f) .
$$

Since $\left\|e^{i(A+i a) t}\right\|=e^{-a t} \rightarrow 0$ as $t \rightarrow \infty$, one gets

$$
\lim _{t \rightarrow \infty} u_{a}(t)=(A+i a)^{-1} f .
$$

Since $f=A y$, one has

$$
\eta(a):=\left\|(A+i a)^{-1} A y-y\right\|=a\left\|(A+i a)^{-1} y\right\| \rightarrow 0 \quad \text { as } a \rightarrow 0 .
$$

The last relation follows from the assumption $y \perp N$. Indeed, by the spectral theorem one has

$$
\begin{aligned}
\lim _{a \rightarrow 0} \eta^{2}(a) & =\lim _{a \rightarrow 0} a^{2}\left\|(A+i a)^{-1} y\right\|^{2}=\lim _{a \rightarrow 0} \int_{-\infty}^{\infty} \frac{a^{2}}{s^{2}+a^{2}} d\left(E_{s} y, y\right) \\
& =\left\|\left(E_{0}-E_{0-0}\right) y\right\|^{2}=\left\|P_{N} y\right\|^{2}=0 .
\end{aligned}
$$

Theorem 1 is proved.

REMARK 1. In a numerical implementation of Theorem 1 one chooses $\tau$ and $a=a(\tau)$, and integrates (2.1) on the interval $[0, \tau]$. One chooses $\tau$ so that

$$
\lim _{\tau \rightarrow \infty}\left\|u_{a(\tau)}-y\right\|=0
$$


In order to choose $a(\tau)$, note that $\left\|u_{a}(t)-u_{a}(\infty)\right\| \leq e^{-a t} / a$, as follows from the derivation of (2.7). Therefore, by Theorem 1, the relation (2.9) holds if

$$
\lim _{\tau \rightarrow \infty} \frac{e^{-a(\tau) \tau}}{a(\tau)}=0, \quad \lim _{\tau \rightarrow \infty} a(\tau)=0 .
$$

For example, one may take $a(\tau)=\tau^{-\gamma}$, where $0<\gamma<1$ is a constant.

Proof of Theorem 2. Let us start with the formula

$$
u_{a, \delta}(t)=\int_{0}^{t} e^{i(A+i a)(t-s)}\left(-i f_{\delta}\right) d s=[i(A+i a)]^{-1}\left(e^{i(A+i a) t}-I\right)\left(i f_{\delta}\right) .
$$

Thus

$$
\mathcal{E}:=\left\|u_{a, \delta}(t)-y\right\| \leq\left\|u_{a, \delta}(t)-u_{a}(t)\right\|+\left\|u_{a}(t)-y\right\|
$$

One has

$$
\left\|u_{a, \delta}(t)-u_{a}(t)\right\| \leq \frac{\left\|f_{\delta}-f\right\|}{a}\left\|I-e^{i A t-a t}\right\| \leq \frac{2 \delta}{a},
$$

and

$$
\left\|u_{a}(t)-y\right\| \leq \frac{e^{-a t}}{a}\|f\|+\eta(a),
$$

where $\eta(a)$ is defined in (2.8), $\lim _{a \rightarrow 0} \eta(a)=0$. Since $\|f\| \leq\left\|f_{\delta}\right\|+\delta \leq c$, one obtains from (2.12)-(2.14):

$$
\lim _{\delta \rightarrow 0} \mathcal{E}=0
$$

provided that $t=t_{\delta}, a=a(\delta)$ and

$$
\lim _{\delta \rightarrow 0} t_{\delta}=\infty, \quad \lim _{\delta \rightarrow 0} a(\delta)=0, \quad \lim _{\delta \rightarrow 0} \frac{e^{-a(\delta) t_{\delta}}}{a(\delta)}=0, \quad \lim _{\delta \rightarrow 0} \frac{\delta}{a(\delta)}=0 .
$$

Theorem 2 is proved.

REMARK 2. There are many choices of $t_{\delta}$ and $a(\delta)$ satisfying relations (2.16). If one has an estimate of the rate of decay of $\eta(a)$ as $a \rightarrow 0$, then one may obtain some rate of convergence of $\mathcal{E}$ to zero as $\delta \rightarrow 0$. However, it is impossible, in general, to get a rate of decay of $\eta(a)$ as $a \rightarrow 0$ without additional assumptions on the data $f$ or on the solution $y$. A typical assumption is $y=A z$, that is, $y \in R(A)$. If fractional powers of $A$ are defined (which is the case when $A \geq 0$, for example) then one may assume $y=A^{\gamma} z, \gamma>0$. Let us show how to get the rate of decay of $\eta(a)$ under such assumptions. Assume, for example, that $y=A z$. Then

$$
\eta^{2}(a)=\int_{-\infty}^{\infty} \frac{a^{2} s^{2}}{a^{2}+s^{2}} d\left(E_{s} z, z\right) \leq a^{2}\|z\|^{2},
$$


and the error bound is

$$
\mathcal{E} \leq \frac{2 \delta}{a}+c_{1} \frac{e^{-a t}}{a}+c_{2} a, \quad c_{2}=\|z\|, \quad c_{1}=\left\|f_{\delta}\right\|+\delta .
$$

Choose, for example, $a=\delta^{\gamma}, 0<\gamma<1$, and $t_{\delta}=\delta^{-\mu}, \mu>\gamma$. Then $\lim _{\delta \rightarrow 0} e^{-a(\delta) t_{\delta}} / a(\delta)=0$ and (2.15) holds with the rate $\delta^{\nu}, \nu=\min (1-\gamma, \gamma)$. If $\gamma=1 / 2$ then $\max _{0<\gamma<1} \min (1-\gamma, \gamma)$ is equal to $1 / 2$, and for $\gamma=1 / 2$ one gets $\mathcal{E}=O\left(\delta^{1 / 2}\right)$ if $t_{\delta}=\delta^{-\mu}$ with $\mu>1 / 2$.

3. Second version of the DSM. Consider problem (2.1) with $a=$ $a(t)$. Let us assume that

$$
0<a(t) \searrow 0, \quad a^{\prime}+a^{2} \in L^{1}(0, \infty), \quad \int_{0}^{\infty} a(s) d s=\infty .
$$

The solution to this problem is

$$
u(t)=\int_{0}^{t} e^{i A(t-s)-\int_{s}^{t} a(p) d p} d s(-i f) .
$$

THEOREM 3. Under the above assumptions one has

$$
\lim _{t \rightarrow \infty}\|u(t)-y\|=0 .
$$

Proof. Since $f=A y$, integrating by parts one gets

$$
u(t)=\left.e^{i A t} e^{-i A s-\int_{s}^{t} a d p} y\right|_{0} ^{t}-\int_{0}^{t} e^{i A(t-s)} a(s) e^{-\int_{s}^{t} a(p) d p} d s y .
$$

Thus

$$
u(t)=y-e^{i A t-\int_{0}^{t} a d p} y-\int_{0}^{t} e^{i A(t-s)} a(s) e^{-\int_{s}^{t} a d p} d s y,
$$

and

$$
\|u(t)-y\| \leq e^{-\int_{0}^{t} a d p}\|y\|+\left\|\int_{0}^{t} e^{i A(t-s)} a(s) e^{-\int_{s}^{t} a d p} d s y\right\|=: J_{1}+J_{2} .
$$

By the last assumption of (3.1) one gets

$$
\lim _{t \rightarrow \infty} J_{1}=0 \text {. }
$$

We now prove that

$$
\lim _{t \rightarrow \infty} J_{2}=0 .
$$

Using the spectral theorem, one gets

$$
J_{2}^{2}=\int_{-\infty}^{\infty} d\left(E_{\lambda} y, y\right)\left|\int_{0}^{t} e^{i \lambda(t-s)} a(s) e^{-\int_{s}^{t} a d p} d s\right|^{2} .
$$


Let us prove that

$$
\lim _{t \rightarrow \infty} \int_{0}^{t} e^{i \lambda(t-s)} a(s) e^{-\int_{s}^{t} a d p} d s=0 \quad \forall \lambda \neq 0 .
$$

From (3.8), (3.9) and the assumption $y \perp N$, the conclusion (3.7) follows.

To verify (3.9), integrating by parts one gets

$$
\begin{aligned}
& J_{3}:=\int_{0}^{t} e^{i \lambda(t-s)} a(s) e^{-\int_{s}^{t} a d p} d s \\
& =\left.\frac{e^{i \lambda(t-s)}}{-i \lambda} a(s) e^{-\int_{s}^{t} a d p}\right|_{0} ^{t}+\frac{1}{i \lambda} \int_{0}^{t} e^{i \lambda(t-s)}\left[a^{\prime}(s)+a^{2}(s)\right] e^{-\int_{s}^{t} a(p) d p} d s .
\end{aligned}
$$

Thus

$$
J_{3}=\frac{a(t)}{-i \lambda}+\frac{e^{i \lambda t-\int_{0}^{t} a d p} a(0)}{i \lambda}+J_{4}, \quad \lambda \neq 0,
$$

where $J_{4}$ denotes the last integral in (3.10). The first two terms in (3.11) tend to zero as $t \rightarrow \infty$ because of the assumptions about $a(t)$.

Assumptions (3.1) imply that

$$
\lim _{t \rightarrow \infty} J_{4}=0 .
$$

Thus, Theorem 3 is proved.

REMARK 3. For example, the function $a(t)=c_{0} /\left(c_{1}+t\right)^{b}$, where $c_{0}, c_{1}>0$ are arbitrary constants and $b \in(1 / 2,1)$ is a constant, satisfies assumptions (3.1).

Let us prove that Theorem 3 yields a stable solution to equation (1.1).

THEOREM 4. There exists a stopping time $t_{\delta}$, with $\lim _{\delta \rightarrow 0} t_{\delta}=\infty$, such that (1.6) holds with $u_{\delta}=u_{\delta}\left(t_{\delta}\right)$, where $u_{\delta}(t)$ is the solution to problem (2.1) with $a=a(t)$ and $f_{\delta}$ in place of $f$, with $\left\|f_{\delta}-f\right\| \leq \delta$.

Proof. One has

$$
\left\|u_{\delta}(t)-y\right\| \leq\left\|u_{\delta}(t)-u(t)\right\|+\|u(t)-y\|,
$$

where $u(t)$ solves problem (2.1) with $a=a(t)$ and exact data. We have proved in Theorem 3 that

$$
\lim _{t \rightarrow \infty}\|u(t)-y\|=0 .
$$

We have

$$
\left\|u_{\delta}(t)-u(t)\right\| \leq \int_{0}^{t} e^{-\int_{s}^{t} a(p) d p} d s\left\|f_{\delta}-f\right\| \leq \frac{\delta}{a(t)} .
$$


Here the estimate $\int_{0}^{t} e^{-\int_{s}^{t} a d p} d s \leq 1 / a(t)$ was used, which is derived easily:

$$
\begin{aligned}
\int_{0}^{t} e^{-\int_{s}^{t} a d p} d s & \leq \frac{1}{a(t)} \int_{0}^{t} a(s) e^{-\int_{s}^{t} a d p} d p=\left.\frac{1}{a(t)} e^{-\int_{s}^{t} a d p}\right|_{0} ^{t} \\
& =\frac{1}{a(t)}\left(1-e^{-\int_{0}^{t} a d p}\right) \leq \frac{1}{a(t)}
\end{aligned}
$$

Choose $t_{\delta}$ so that

$$
\lim _{\delta \rightarrow 0} t_{\delta}=\infty, \quad \lim _{\delta \rightarrow 0} \frac{\delta}{a\left(t_{\delta}\right)}=0 .
$$

This is obviously possible. Then (3.13)-(3.15) imply

$$
\lim _{\delta \rightarrow 0}\left\|u_{\delta}\left(t_{\delta}\right)-y\right\|=0 .
$$

Theorem 4 is proved.

\section{Third version of DSM for solving equation (1.1)}

$$
\dot{u}=-u+T_{a(t)}^{-1} A^{*} f, \quad u(0)=0,
$$

where $f=A y, y \perp N$, and $a(t)>0$ is a monotonically decaying continuous function such that $\lim _{t \rightarrow \infty} a(t)=0$ and $\int_{0}^{\infty} a(t) d t=\infty$. We could take the initial condition $u(0)=u_{0}$ arbitrary. The contribution to the solution of problem (4.1) which comes from the initial condition $u_{0}$ is the term $u_{0} e^{-t}$. It decays exponentially fast and our arguments do not depend on this term essentially. To simplify and shorten our argument we take $u_{0}=0$.

THEOREM 5. Under the assumptions of Theorem 3 the solution $u(t)$ to problem (4.1) exists, is unique, is defined for all $t \geq 0$, and $\lim _{t \rightarrow \infty} u(t)=y$, where $y$ is the minimal-norm solution to (1.1).

Proof. One has

$$
u(t)=\int_{0}^{t} e^{-(t-s)} T_{a(s)}^{-1} A^{*} A y d s=\int_{0}^{t} e^{-(t-s)} y d s-\int_{0}^{t} e^{-(t-s)} a(s) T_{a(s)}^{-1} y d s .
$$

Thus

$$
\|u(t)-y\| \leq e^{-t}\|y\|+\int_{0}^{t} e^{-(t-s)} a(s)\left\|T_{a(s)}^{-1} y\right\| d s .
$$

One can easily check that if $b(s)$ is a continuous function on $[0, \infty)$ and $b(\infty)=\lim _{s \rightarrow \infty} b(s)$ exists, then

$$
\lim _{t \rightarrow \infty} \int_{0}^{t} e^{-(t-s)} b(s) d s=b(\infty)
$$


Thus, Theorem 5 will be proved if one checks that

$$
\lim _{a \rightarrow 0} a\left\|T_{a}^{-1} y\right\|=0 \quad \forall y \in H .
$$

To prove (4.4) one writes, using the spectral theorem,

$$
a^{2}\left\|T_{a}^{-1} y\right\|^{2}=\int_{0}^{\infty} \frac{a^{2}}{(s+a)^{2}} d\left(E_{s} y, y\right)=\left\|P_{N} y\right\|^{2}=0 .
$$

Theorem 5 is proved.

Let us prove that the DSM method (4.1) yields a stable solution to problem (1.1). Assume that $f$ is replaced by $f_{\delta}$, with $\left\|f_{\delta}-f\right\| \leq \delta$, in equation (4.1), and denote by $u_{\delta}(t)$ the corresponding solution. Then, using the estimate (3.13), one gets

$$
\lim _{\delta \rightarrow 0}\left\|u_{\delta}\left(t_{\delta}\right)-y\right\|=0
$$

provided that

$$
\lim _{\delta \rightarrow 0} t_{\delta}=\infty, \quad \lim _{\delta \rightarrow 0} \frac{\delta}{\sqrt{a\left(t_{\delta}\right)}}=0 .
$$

To check the sufficiency of the second condition of (4.7) for (4.6) to hold, one proceeds as follows:

$$
\begin{aligned}
\left\|u_{\delta}(t)-u(t)\right\|= & \left\|\int_{0}^{t} e^{-(t-s)} T_{a(s)}^{-1} A^{*}\left(f_{\delta}-f\right) d s\right\| \\
& \leq \delta \int_{0}^{t} e^{-(t-s)} \frac{1}{2 \sqrt{a(s)}} d s \leq \frac{\delta}{2 \sqrt{a(t)}} .
\end{aligned}
$$

Here we have used the monotonicity of $a(t)$, which implies $a(t) \leq a(s)$ if $t \geq s$, and the estimate $\left\|T_{a}^{-1} A^{*}\right\| \leq 1 /(2 \sqrt{a})$, which was proved earlier.

Let us state the result we have proved.

THEOREM 6. If $t_{\delta}$ is chosen so that (4.7) holds, then the solution $u_{\delta}(t)$ to problem (4.1) with noisy data $f_{\delta}$ in place of $f$ satisfies (4.6).

5. A new discrepancy principle. The usual discrepancy principle is described in the introduction. It requires solving nonlinear equation $\left\|A u_{a, \delta}-f\right\|=C \delta$ with $C=$ const, $1<C<2$, where $u_{a, \delta}=T_{a}^{-1} A^{*} f_{\delta}$. Thus one has to know the exact minimizer $u_{a, \delta}$ of the functional $F(u)=$ $\left\|A u-f_{\delta}\right\|^{2}+a\|u\|^{2}$, or the exact solution of the equation $T_{a} u=A^{*} f_{\delta}$.

In this section we discuss the following question:

How does one formulate the discrepancy principle in the case when $u_{a, \delta}$ is not the exact solution of the minimization problem $F(u)=$ min, but an approximate solution? 
Let us state the result.

TheOREM 7. Assume that $A$ is a bounded linear operator in a Hilbert space $H$, that $f=A y, y \perp N,\left\|f_{\delta}-f\right\| \leq \delta,\left\|f_{\delta}\right\|>C \delta, C=$ const, $C \in(1,2)$, and $u_{a, \delta}$ is any element which satisfies the inequality

$$
F\left(u_{a, \delta}\right) \leq m+\left(C^{2}-1-b\right) \delta^{2},
$$

where

$$
F(u)=\left\|A u-f_{\delta}\right\|^{2}+a\|u\|^{2}, \quad m=\inf _{u \in H} F(u),
$$

$b=$ const $>0$ and $C^{2}>1+b$. Then the equation

$$
\left\|A u_{a, \delta}-f_{\delta}\right\|=C \delta
$$

has a solution for any fixed $\delta>0$, with $\lim _{\delta \rightarrow 0} a(\delta)=0$, and

$$
\lim _{\delta \rightarrow 0}\left\|u_{\delta}-y\right\|=0
$$

where $u_{\delta}=u_{a(\delta), \delta}$ and $a(\delta)$ solves (5.3).

Proof. To prove the existence of a solution to (5.3), we denote $\left\|A u_{a, \delta}-f_{\delta}\right\|$ by $h(\delta, a)$, check that $h(\delta,+0)<C \delta, h(\delta, \infty)>C \delta$, and note that $h(\delta, a)$ is a continuous function of $a$ on the interval $(0, \infty)$. This implies the existence of a solution $a=a(\delta)$ to equation (5.3).

As $a \rightarrow \infty$, one has

$$
a\left\|u_{a, \delta}\right\|^{2} \leq F\left(u_{a, \delta}\right) \leq m+\left(C^{2}-1-b\right) \delta^{2} \leq F(0)+\left(C^{2}-1-b\right) \delta^{2},
$$

so, with $c:=F(0)+\left(C^{2}-1-b\right) \delta^{2}$, one obtains

$$
\left\|u_{a, \delta}\right\| \leq c / \sqrt{a} \rightarrow 0 \quad \text { as } a \rightarrow \infty \text {. }
$$

Thus

$$
h(\delta, \infty)=\left\|A 0-f_{\delta}\right\|=\left\|f_{\delta}\right\|>C \delta .
$$

As $a \rightarrow 0$, one has

$$
h^{2}(\delta, a) \leq F\left(u_{a, \delta}\right) \leq m+\left(C^{2}-1-b\right) \delta^{2} \leq F(y)+\left(C^{2}-1-b\right) \delta^{2} .
$$

Since

$$
F(y)=\delta^{2}+a\|y\|^{2},
$$

one has

$$
h^{2}(\delta, a) \leq\left(C^{2}-b\right) \delta^{2}+a\|y\|^{2},
$$

and

$$
h(\delta,+0) \leq\left(C^{2}-b\right)^{1 / 2} \delta<C \delta .
$$

Finally, the continuity of $h(\delta, a)$ with respect to $a \in(0, \infty)$ for any fixed $\delta>0$ follows from the continuity of the bounded operator $A$ and the continuity of $u_{a, \delta}$ with respect to $a \in(0, \infty)$. Thus, the existence of a solution $a=a(\delta)>0$ of equation (5.3) is proved. One takes a solution for which $\lim _{\delta \rightarrow 0} a(\delta)=0$. 
Such a solution exists because $h(\delta,+0)$ and $m=m(\delta, a)$ tend to zero as $\delta \rightarrow 0$ and $a \rightarrow 0$.

Let us prove (5.4). One has

$$
\begin{aligned}
F\left(u_{\delta}\right) & =\left\|A u_{\delta}-f_{\delta}\right\|^{2}+a(\delta)\left\|u_{\delta}\right\|^{2} \leq\left\|A y-f_{\delta}\right\|^{2}+a(\delta)\|y\|^{2} \\
& \leq \delta^{2}+a(\delta)\|y\|^{2} .
\end{aligned}
$$

Since $\left\|A u_{\delta}-f_{\delta}\right\|=C \delta, C>1$ it follows from (5.7) that

$$
\left\|u_{\delta}\right\| \leq\|y\| \text {. }
$$

Thus one may assume that $u_{\delta} \rightarrow u$ as $\delta \rightarrow 0$.

Let us prove that $A u=f$. First, we observe that

$$
\left\|A u_{\delta}-f\right\| \leq\left\|A u_{\delta}-f_{\delta}\right\|+\left\|f_{\delta}-f\right\| \leq C \delta+\delta,
$$

so

$$
\lim _{\delta \rightarrow 0}\left\|A u_{\delta}-f\right\|=0 .
$$

Secondly, for any $v \in H$ we have

$$
(f-A u, v)=\lim _{\delta \rightarrow 0}\left(A u_{\delta}-A u, v\right)=\lim _{\delta \rightarrow 0}\left(u_{\delta}-u, A^{*} v\right)=0,
$$

because $u_{\delta} \rightarrow u$. Since $v$ is arbitrary, one concludes from (5.10) that $A u=f$. From (5.8) it follows that $\|u\| \leq\|y\|$. As the minimal-norm solution to the equation $A u=f$ is unique, one obtains $u=y$. Thus, $u_{\delta} \rightarrow y$ and $\left\|u_{\delta}\right\| \leq\|y\|$. This implies (5.4), as follows from (1.18).

Theorem 7 is proved.

6. Discrepancy principle does not yield uniform convergence with respect to the data. In this section we make the following assumption.

Assumption A. A is a linear bounded operator in a Hilbert space $H$, $N:=N(A)=N\left(A^{*}\right):=N^{*}=\{0\}, A^{-1}$ is unbounded, $A y=f,\left\|f_{\delta}-f\right\| \leq \delta$, $\left\|f_{\delta}\right\|>\delta$.

Let $a=a(\delta)$ be chosen by the discrepancy principle,

$$
\left\|A u_{a, \delta}-f_{\delta}\right\|=C \delta, \quad u_{\delta}=u_{a(\delta), \delta}=T_{a(\delta)}^{-1} A^{*} f_{\delta} .
$$

Consider the set

$$
S_{\delta}:=\left\{v:\left\|A v-f_{\delta}\right\| \leq \delta\right\} .
$$

We are interested in the following question: given $\left\{f_{\delta}\right\}_{\delta \in\left(0, \delta_{0}\right)}$, where $\delta_{0}>0$ is a small number, and assuming that $a(\delta)$ is the solution to (6.1), can one guarantee uniform convergence with respect to the data $f$ ?

In other words, is it true that

$$
\lim _{\delta \rightarrow 0} \sup _{v \in S_{\delta}}\left\|u_{\delta}-v\right\|=0 ?
$$

The answer is no. 
THEOREM 8. There exist $f_{\delta}$ such that

$$
\lim _{\delta \rightarrow 0} \sup _{v \in S_{\delta}}\left\|u_{\delta}-v\right\| \geq c>0, \quad c=\text { const. }
$$

Proof. Set $T_{a}^{-1} A^{*}=: G, u_{\delta}=G f_{\delta},\|G\|=1 /(2 \sqrt{a})$. We have proved in (1.10) that $\|G\| \leq 1 / 2 \sqrt{a}$, but in fact equality holds because in (1.10), $U$ is unitary under Assumption A. Thus, one can find an element $p=p_{a}$ with $\|p\|=\delta / 2$ such that

$$
\|G p\| \geq \frac{1}{2}\|G\|\|p\|=\frac{\delta}{8 \sqrt{a}} .
$$

Assumption A implies that the ranges $R(A)$ and $R(T)$ are dense in $H$. Thus one can find an element $z=z_{a, \delta}$ such that

$$
\left\|f_{\delta}-A T^{b} z-p\right\| \leq \delta / 8, \quad b \in(0,1), b=\text { const. }
$$

For any $v$ one has

$$
\left\|G f_{\delta}-v\right\| \leq\left\|G f_{\delta}-G A v\right\|+\|G A v-v\| .
$$

Take $v=T^{b} z$ and let $M>0$ be an arbitrarily large fixed constant. Then

$$
\lim _{\delta \rightarrow 0} \sup _{v \in S_{\delta}, v=B^{b} z,\|z\| \leq M}\|G A v-v\|=0,
$$

because

$$
\|G A v-v\|=\left\|T_{a}^{-1} T v-v\right\|=\left\|-a T^{-1} T^{b} z\right\|=\sup _{s \geq 0} \frac{s^{b}}{s+a}=c a^{b},
$$

where $c=b^{b}(1-b)^{1-b}$.

From (6.7) and (6.6) one sees that

$$
\lim _{\delta \rightarrow 0} \sup _{v \in S_{\delta}, v=T^{b} z,\|z\| \leq M}\left\|G f_{\delta}-v\right\|=0
$$

if and only if

$$
\lim _{\delta \rightarrow 0} \sup _{v \in S_{\delta}, v=T^{b} z,\|z\| \leq M}\left\|G f_{\delta}-G A v\right\|=0 .
$$

Take $z=z_{a}, v=T^{b} z$. Then

$$
\left\|f_{\delta}-A v\right\| \leq\|p\|+\left\|f_{\delta}-A B^{b} z-p\right\| \leq \delta / 2+\delta / 8=5 \delta / 8 \leq \delta,
$$

and, using (6.5), one gets

$$
\begin{aligned}
\left\|G f_{\delta}-G A v\right\| & =\left\|G\left(f_{\delta}-A v-p\right)+G p\right\| \geq\|G p\|-\|G\| \frac{\delta}{8} \\
& \geq \frac{\delta}{\sqrt{a}}\left(\frac{1}{8}-\frac{1}{16}\right)=\frac{\delta}{16 \sqrt{a}} .
\end{aligned}
$$

If $\delta / \sqrt{a} \geq c>0$, then, according to (6.12), (6.10) fails.

Let us find $f_{\delta}$ such that for $a=a(\delta)$, defined by the discrepancy principle, one has $\delta / \sqrt{a} \geq c>0$. This will complete the proof of Theorem 8 . Let us 
assume for simplicity that $A=A^{*}>0$ is compact. Then $T=A^{*} A=A^{2}$, and equation (6.1) becomes

$$
\begin{aligned}
C^{2} \delta^{2} & =\left\|\left[A\left(A^{2}+a\right)^{-1} A-I\right] f_{\delta}\right\|^{2} \\
& =\sum_{j=1}^{\infty}\left[\frac{\lambda_{j}^{2}}{\lambda_{j}^{2}+a}-1\right]^{2}\left|f_{\delta j}\right|^{2}=\sum_{j=1}^{\infty} \frac{a^{2}\left|f_{\delta j}\right|^{2}}{\left(\lambda_{j}+a\right)^{2}} .
\end{aligned}
$$

Here $\lambda_{j}$ are the eigenvalues of $A^{2}, f_{\delta j}=\left(f_{\delta}, \varphi_{j}\right), A^{2} \varphi_{j}=\lambda_{j} \varphi_{j},\left\|\varphi_{j}\right\|=1$. Assume, for example, that $\lambda_{j}=1 / j$ and $\left|f_{\delta j}\right|^{2}=1 / j^{2}$. Then (6.13) becomes

$$
C^{2} \frac{\delta^{2}}{a^{2}}=\sum_{j=1}^{\infty} \frac{j^{-2}}{\left(j^{-1}+a\right)^{2}}=: I(a) .
$$

Note that

$$
I(a) \sim I_{1}(a) \quad \text { as } a \rightarrow 0
$$

where

$$
I_{1}(a):=\int_{1}^{\infty} \frac{x^{-2}}{\left(x^{-1}+a\right)^{2}} d x .
$$

One has

$$
\begin{aligned}
I_{1}(a) & =\int_{1}^{\infty} \frac{x^{-2}}{\left(x^{-1}+a\right)^{2}} d x=\int_{0}^{1} \frac{d s}{(s+a)^{2}}=-\left.(s+a)^{-1}\right|_{0} ^{1} \\
& =a^{-1}\left[1-\frac{a}{a+2}\right]=\frac{1}{a}[1+O(a)], \quad a \rightarrow 0 .
\end{aligned}
$$

This and (6.14) imply $\delta / \sqrt{a} \geq c>0$ as $\delta \rightarrow 0$. Theorem 8 is proved.

7. Iterative processes for solving equation (1.1). In this section convergent iterative processes for solving equation (1.1) are constructed in the case when $A$ is a closed, densely defined, unbounded operator in $H$. Consider the process

$$
u_{n+1}=B u_{n}+T_{a}^{-1} A^{*} f, \quad u_{1} \perp N, B:=a T_{a}^{-1},
$$

where $a=$ const $>0$ and the initial element $u_{1}$ is arbitrary in the subspace $N^{\perp}$, where $N:=N(A)=N(T), T=A^{*} A, T_{a}=T+a I$. Note that $B \geq 0$ and $\|B\| \leq 1$.

THEOREM 9. Under the above assumptions one has

$$
\lim _{n \rightarrow \infty}\left\|u_{n}-y\right\|=0 .
$$

Proof. Let $w_{n}=u_{n}-y$. Then

$$
w_{n+1}=B w_{n}=B^{n} w, \quad w:=u_{1}-y, \quad w \perp N .
$$


Let us prove that

$$
\lim _{n \rightarrow \infty}\left\|B^{n} w\right\|=0 .
$$

If (7.4) is verified, then Theorem 9 is proved. We have

$$
\left\|B^{n} w\right\|^{2}=\int_{0}^{\infty} \frac{a^{2 n}}{(a+s)^{2 n}} d\left(E_{s} w, w\right)=\int_{s>b}+\int_{0 \leq s \leq b}=: J_{1}+J_{2},
$$

where $E_{s}$ is the resolution of the identity corresponding to the operator $T \geq 0$, and $b>0$ is a small number which will be chosen later. For any fixed $b>0$ one has $\lim _{n \rightarrow \infty} J_{1}=0$ because $a /(a+s) \leq a /(a+b)<1$ if $s \geq b$. On the other hand, $J_{2} \leq \int_{0}^{b} d\left(E_{s} w, w\right)$, and $\lim _{b \rightarrow 0} \int_{0}^{b} d\left(E_{s} w, w\right)=0$ because $w \perp N=E_{0} H$. Therefore, given an arbitrary small number $\eta>0$ one can choose $b>0$ such that $J_{2} \leq \eta / 2$. Fix such a $b$ and choose $n$ sufficiently large so that $J_{1} \leq \eta / 2$. Then $\left\|B^{n} w\right\|^{2} \leq \eta$. Since $\eta$ is arbitrarily small, we have proved (7.4). Theorem 9 is proved.

REMARK 4. The iterative process (7.1) yields a stable solution of equation (1.1). Indeed, let $f_{\delta}$ be given with $\left\|f_{\delta}-f\right\| \leq \delta$, and let $u_{n, \delta}$ be defined by (7.1) with $f_{\delta}$ in place of $f$. Let $w_{n, \delta}=u_{n, \delta}-y$. Then

$$
w_{n+1, \delta}=B w_{n, \delta}+T_{a}^{-1} A^{*}\left(f_{\delta}-f\right),
$$

SO

$$
w_{n+1, \delta}=\sum_{j=1}^{n} B^{j} T^{-1} A^{*}\left(f_{\delta}-f\right)+B^{n}\left(u_{1}-y\right), \quad\left(u_{1}-y\right) \perp N .
$$

We have proved above that

$$
\left\|B^{n}\left(u_{1}-y\right)\right\|=: \mathcal{E}(n) \rightarrow 0 \quad \text { as } n \rightarrow \infty .
$$

One has

$$
\left\|\sum_{j=0}^{n} B^{j} T_{a}^{-1} A^{*}\left(f_{\delta}-f\right)\right\| \leq \frac{(n+1) \delta}{2 \sqrt{a}},
$$

because $\|B\| \leq 1$ and $\left\|T_{a}^{-1} A^{*}\right\| \leq 1 /(2 \sqrt{a})$. From (7.7) and (7.8) one finds the stopping rule, i.e., the number $n(\delta)$ such that $\lim _{\delta \rightarrow 0}\left\|w_{n(\delta), \delta}\right\|=0$. This $n(\delta)$ is found for any fixed small $\delta$ as the minimizer for the problem

$$
\frac{(n+1) \delta}{2 \sqrt{a}}+\mathcal{E}(n)=\min .
$$

Alternatively, one can find $n_{1}(\delta)$ from the equation

$$
\mathcal{E}(n)=\frac{(n+1) \delta}{2 \sqrt{a}} .
$$

Clearly $n(\delta)$ and $n_{1}(\delta)$ tend to $\infty$ as $\delta \rightarrow 0$. 
8. Discrepancy principle for DSM. In this section we formulate and justify a discrepancy principle for DSM.

Let us start with the version (4.1). We assume that $a(t)>0$ is a monotonically decaying twice continuously differentiable function, $\lim _{t \rightarrow \infty}[a(t)+|\dot{a}|$ $+\ddot{a}]=0, \ddot{a}>0$, and $\lim _{t \rightarrow \infty} \dot{a}(t) / a(t)=0$, for example, $a(t)=c_{1} /\left(c_{0}+t\right)^{b}$, where $c_{1}, c_{0}$ and $b$ are positive constants, with $b \in(0.5,1)$. For this $a(t)$ all the assumptions (3.1) hold.

Theorem 10. The equation

$$
\left\|A T_{a(t)}^{-1} A^{*} f_{\delta}-f_{\delta}\right\|=C \delta, \quad C=\text { const, } 1<C<2,
$$

has a solution $t=t_{\delta}$, with $\lim _{\delta \rightarrow 0} t_{\delta}=\infty$, such that (4.6) holds, where $u_{\delta}(t)$ is the solution to (4.1) with $f_{\delta}$ in place of $f$, and $\left\|f_{\delta}\right\|>C \delta$.

Proof. We have proved earlier that equation (8.1) has a unique solution $a=a_{\delta}$ and $\lim _{\delta \rightarrow 0} a_{\delta}=0$. If $a(t)$ is a monotonically decaying function such that $\lim _{t \rightarrow \infty} a(t)=0$, then the equation $a_{\delta}=a(t)$ uniquely defines $t=t_{\delta}$ such that $a\left(t_{\delta}\right)=a_{\delta}$, and $\lim _{\delta \rightarrow 0} t_{\delta}=\infty$.

Let us sketch the proof of (4.6), where $u_{\delta}\left(t_{\delta}\right)=\int_{0}^{t_{\delta}} e^{-\left(t_{\delta}-s\right)} T_{a(s)}^{-1} A^{*} f_{\delta} d s$ and $t_{\delta} \rightarrow \infty$ as $\delta \rightarrow 0$. We have proved (cf. (1.17)) that $\left\|T_{a\left(t_{\delta}\right)}^{-1} A^{*} f_{\delta}\right\| \leq\|y\|$, and

$$
\lim _{\delta \rightarrow 0}\left\|T_{a\left(t_{\delta}\right)}^{-1} A^{*} f_{\delta}-y\right\|=0 .
$$

(cf (1.18)). It is clear that $\lim _{t \rightarrow \infty} \int_{0}^{t} e^{-(t-s)} g(s) d s=g(\infty)$ provided that $g$ is a continuous function and $g(\infty):=\lim _{t \rightarrow \infty} g(t)$ exists.

Note that $\lim _{s \rightarrow t_{\delta}}\left\|T_{a\left(t_{\delta}\right)}^{-1} A^{*} f_{\delta}-T_{a(s)}^{-1} A^{*} f_{\delta}\right\|=0$. We have

$$
\int_{0}^{t_{\delta}} e^{-\left(t_{\delta}-s\right)} T_{a(s)}^{-1} A^{*} f_{\delta} d s=y+o(1) \quad \text { as } \delta \rightarrow 0 .
$$

To check this we use equation (8.2), the formula

$$
T_{a\left(t_{\delta}\right)}^{-1}-T_{a(s)}^{-1}=T_{a(s)}^{-1}\left[a\left(t_{\delta}\right)-a(s)\right] T_{a\left(t_{\delta}\right)}^{-1},
$$

the estimates $\left\|T_{a\left(t_{\delta}\right)}^{-1} A^{*} f_{\delta}\right\| \leq\|y\|$ and $\left\|T_{a(s)}^{-1}\right\| \leq 1 / a(s)$, and the relation

$$
\lim _{t \rightarrow \infty} \int_{0}^{t} e^{-(t-s)} \frac{a(s)-a(t)}{a(s)} d s=0,
$$

which holds due to our assumptions on $a(t)$. Theorem 10 is proved.

\section{References}

[1] M. Gavurin, Nonlinear functional equations and continuous analogs of iterative methods, Izv. Vyssh. Uchebn. Zaved. Mat. 5 (1958), 18-31 (in Russian). 
[2] V. Ivanov, V. Vasin and V. Tanana, Theory of Linear Ill-Posed Problems, Nauka, Moscow, 1978 (in Russian).

[3] T. Kato, Perturbation Theory for Linear Operators, Springer, New York, 1984.

[4] O. Liskovets, Regularization of equations with a closed linear operator, Differential Equations 6 (1970), 972-976.

[5] V. Morozov, Methods of Solving Incorrectly Posed Problems, Springer, New York, 1984.

[6] D. Phillips, A technique for numerical solution of certain integral equations of the first kind, J. Assoc. Comput. Mach. 9 (1962), 84-97.

[7] A. G. Ramm, Inverse Problems, Springer, New York, 2005.

[8] —, Dynamical Systems Method for Solving Operator Equations, Elsevier, Amsterdam, 2007

[9] —, On the discrepancy principle, Nonlinear Funct. Anal. Appl. 8 (2003), 307-312.

[10] —, Discrepancy principle for the dynamical systems method, Comm. Nonlinear Sci. Numer. Simulation 10 (2005), 95-101.

[11] —, A new discrepancy principle, J. Math. Anal. Appl. 310 (2005), 342-345.

[12] -, Dynamical systems method for solving nonlinear operator equations, Int. J. Appl. Math. Sci. 1 (2004), 97-110.

[13] -, Dynamical systems method for solving operator equations, Comm. Nonlinear Sci. Numer. Simulation 9 (2004), 383-402.

[14] - Inequalities for solutions to some nonlinear equations, Nonlinear Funct. Anal. Appl. 9 (2004), 233-243.

[15] —, Dynamical systems method and surjectivity of nonlinear maps, Comm. Nonlinear Sci. Numer. Simulation 10 (2005), 931-934.

[16] —, DSM for ill-posed equations with monotone operators, ibid. 10 (2005), 935-940.

[17] —, Dynamical systems method (DSM) and nonlinear problems, in: Spectral Theory and Nonlinear Analysis, J. Lopez-Gomez (ed.), World Sci., Singapore, 2005, 201228.

[18] - Dynamical systems method for nonlinear equations in Banach spaces, Comm. Nonlinear Sci. Numer. Simulation 11 (2006), 306-310.

[19] —, Dynamical systems method and a homeomorphism theorem, Amer. Math. Monthly 113 (2006), 928-933.

[20] - Dynamical systems method (DSM) for unbounded operators, Proc. Amer. Math. Soc. 134 (2006), 1059-1063.

[21] —, Dynamical systems method (DSM) for selfadjoint operators, J. Math. Anal. Appl. 328 (2007), 1290-1296.

[22] —, On unbounded operators and applications, Appl. Math. Lett. 21 (2008), 377-382.

[23] —, Ill-posed problems with unbounded operators, J. Math. Anal. Appl. 325 (2007), 490-495.

[24] —, Two results on ill-posed problems, Int. J. Appl. Math. Statist. 11 (2007), 136-139.

[25] - Iterative solution of linear equations with unbounded operators, J. Math. Anal. Appl. 330 (2007), 1338-1346.

[26] - On a new notion of regularizer, J. Phys. A 36 (2003), 2191-2195.

[27] —, Linear ill-posed problems and dynamical systems, J. Math. Anal. Appl. 258 (2001), 448-456.

[28] -, Regularization of ill-posed problems with unbounded operators, ibid. 271 (2002), $547-550$.

[29] —, A DSM proof of surjectivity of monotone nonlinear mappings, Ann. Polon. Math. 95 (2008), 135-139.

[30] —, DSM for general nonlinear equations, Nonlinear Anal. 69 (2008), 1934-1940. 
[31] A. G. Ramm, Discrepancy principle for DSM II, Comm. Nonlinear Sci. Numer. Simulation 13 (2008), 1256-1263.

[32] A. F. Ramm and N. S. Hoang, Solving ill-conditioned linear algebraic systems by the dynamical systems method, Inverse Problems Sci. Engrg. 16 (2008), 617-630.

[33] —, - Dynamical systems method for solving linear finite-rank operator equations, Ann. Polon. Math. 95 (2009), 77-93.

[34] -, - A new version of the Dynamical Systems Method (DSM) for solving nonlinear equations with monotone operators, Differential Equations Appl. 1 (2009), 1-25.

[35] —, -, A nonlinear inequality, J. Math. Inequal. 2 (2008), 459-464.

[36] - - - Dynamical Systems Gradient method for solving nonlinear equations with monotone operators, Acta Appl. Math., to appear; doi 10.1007/s10440-008-9308-1.

[37] —, - An iterative scheme for solving nonlinear equations with monotone operators, BIT 48 (2008), 725-741.

[38] —, - A discrepancy principle for equations with monotone continuous operators, Nonlinear Anal., to appear; doi 10.1016/j.na.2008.09.014.

[39] A. Tikhonov, A. Leonov and A. Yagola, Nonlinear Ill-Posed Problems, Chapman and Hall, London, 1998.

Mathematics Department

Kansas State University

Manhattan, KS 66506-2602, U.S.A.

E-mail: ramm@math.ksu.edu

Received 12.8.2008

and in final form 17.11.2008 